\title{
String theory under scrutiny
}

One of the main charges

against string theory is that it cannot make specific predictions that may be checked against

an experiment. But as

Nancy Cartwright and

Roman Frigg explain, other

criteria should be taken into account too when evaluating scientific research

Ever since antiquity, attempts have been made to reduce an apparently complex reality to a few elementary building blocks from which everything else is constructed. This project - now called reductionism - has a long history of failures. One example is the 200 -year-long attempt to describe all physical processes in terms of mechanics, such as James Clerk Maxwell's mechanical models of the electromagnetic field. Another is Hermann Weyl's failed attempt to unify electromagnetism and gravity in a single theory shortly after Einstein had introduced special relativity.

However, reductionism has achieved some notable successes too, and these have spurred on the search for unity in theoretical physics. The Standard Model of particle physics, which emerged in the 1970 s and describes the electromagnetic, strong and weak forces in a single framework, is a major triumph in this regard. But as the Standard Model says nothing about the fourth force of nature-gravity-many physicists believe that the end point of unification has not yet been reached. String theory is currently the dominant research programme pursuing this quest.

Although dominant, string theory is not free from controversy. Critics, one of the most prominent being Lee Smolin of the Perimeter Institute in Canada, take the theory to task for not having produced a single new prediction that would allow it to be compared with experiment. They claim that the features of string theory that are at least potentially testable, such as the existence of supersymmetry and cosmic strings, are not specific to string theory. In addition, those features that are specific to string theory, first and foremost the existence of strings, either do not lead to precise predictions or lead to predictions that are impossible to test with current technology.

This, argue Smolin and other critics, is un-

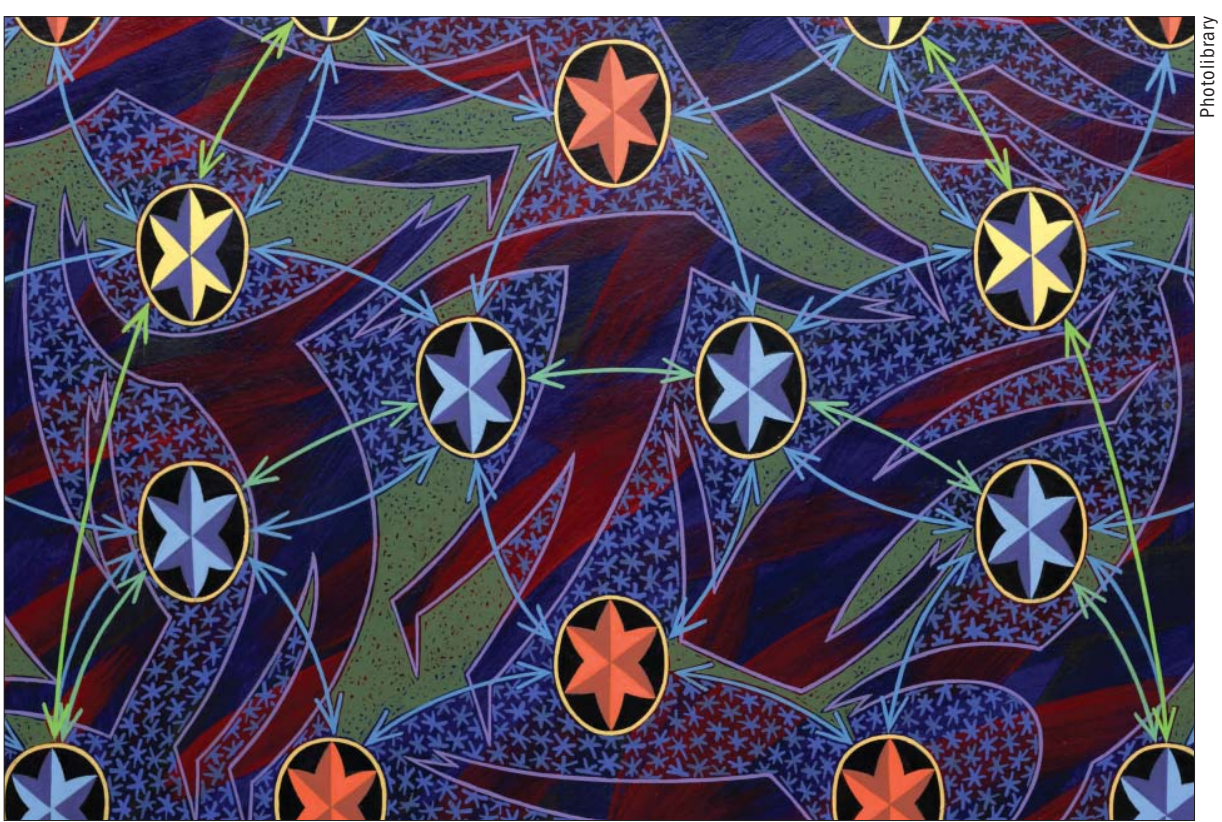

Measuring up String theory can unify and explain - but how far has it progressed in other regards?

acceptable because a scientific theory must stand up to experimental scrutiny. Hence, string theory must either undergo a fundamental change or else it has to be given up. But how sound a judge of a scientific theory is testability, and is it strong enough to rule out string theory as a viable physical theory?

\section{Positivist evolution}

This emphasis on prediction and experimentation is reminiscent of the philosophy of science of Karl Popper and the positivists of the Vienna Circle (who included Moritz Schlick, Rudolf Carnap and Otto Neurath) and the Berlin School, which grew up round Hans Reichenbach. In the 1920s and 1930s these philosophers, following the tradition of Ernst Mach and other empiricists, suggested that we should only accept theories that can be compared with reality in experiments. Any domain of investigation that cannot comply with these standards should thus

\section{String theory's failure to make testable} predictions leaves us with no reason to believe that it gives us a true picture be rejected as unscientific.

This view of science was widely accepted until the 1960s, when various philosophers most notably Imre Lakatos, Thomas Kuhn and Paul Feyerabend - pointed out that it was unduly restrictive, especially when it comes to new theories. Since new theories are not as fully developed as long-standing ones, they may well be sketchy in how they deal with phenomena that are well treated by older theories. Indeed, a new theory may even get some of its predictions wrong. Yet if we throw new theories out on such grounds, or because they are not sufficiently precise, we risk being stuck with the same old physics, perhaps forever.

For these reasons Lakatos proposed that the object of evaluation should not be an individual theory as viewed at one particular time but rather the larger "research programme" that spawns that theory. A research programme is characterized by a core set of ideas, techniques, rules and assumptions, and theories are expected to evolve in accord with these. Good research programmes are those that are progressive, i.e. those whose theories get better and better, even if individual theories face serious difficulties at certain times.

Newton's theory of gravity is a good example of a progressive research programme. When it was first proposed, the theory had only limited empirical success and faced a host of anomalies. But Newton and his followers eliminated one anomaly after another, with the theory then triumphing for over 200 years before Einstein's general 
theory of relativity superseded it. For instance, Newtonian gravity made incorrect predictions for the orbit of Uranus; but instead of regarding this as a refutation of the theory, physicists made the auxiliary assumption that Uranus' motion was influenced by the gravitational force of a new, then unobserved, planet. A few years later, this planet - Neptune - was indeed discovered. Had the Newtonians adopted the strict testability view of science, one of the most powerful theories in physics would never have got off the ground.

\section{How to progress}

The crucial question for string theory is thus not whether the theory in its current form can be tested, but whether the research programme of string theory is progressive. A research programme can progress in many different ways corresponding to different virtues that a good scientific theory is supposed to have. These include the following (which are not specific to Lakatos' philosophy): having a large range of varied empirical applications; generating successful novel predictions; spawning new technologies; answering perplexing problems; consistency; elegance; simplicity; explanatory power; unifying power; and, last but not least, truth.

Radical string critics would then conclude that string theory is progressive only in the dimensions of elegance and simplicity (in the sense that the theory contains only one class of basic objects - strings - from which all the basic particles and forces follow), while being largely stagnant in the other dimensions. However, because string theory requires the gravitational force to exist, it represents an important step towards a unified theory of gravity and quantum mechanics. String theory has also had some success as a tool to study quark-gluon plasmas in energy regimes that are difficult to address using existing theoretical techniques (see "Stringscape" on pages 35-47). These two achievements suggest that string theory shows at least some signs of progress in the dimensions of unifying and explanatory power, respectively.

Nevertheless, a research programme that progresses only in some dimensions, while being by and large stagnant in the others, surely does not count as being progressive. Contrasting string theory with Maxwell's unification of electricity and magnetism, for example, we can see that the latter was genuinely progressing and eventually successful in every dimension. It used the new and powerful concept of a field, which made the theory simple and elegant, while at the same time giving rise to a whole set of new phenomena that led to new predictions. The most astonishing of these was that electromagnetic waves were light - an unexpected result that led to the discovery of the radio, infrared, ultraviolet and other waves that we now view as ubiquitous.

Sceptics might say that by taking the ana-

\section{Truth in physics}

Physicists generally hold contradictory beliefs about the role of truth in science. On the one hand there is the rhetoric about truth: that science is all about trying to uncover how the world really is. But on the other hand, physicists often violently resist foundational programmes that try to figure out what the world would have to be like if a theory were true. For example, rather than trying to understand deep truths about the world that could be lurking in issues in the foundations of quantum mechanics, such as the measurement problem, most physicists are eventually interested only in deriving observational predictions - even though their "sales talk" promises the contrary. However, when stripped of such "truth-talk", the focus on prediction and application is not an illegitimate attitude. Indeed, there is vivid controversy in the philosophy of science over the question of whether truth really is a justifiable aim for science, or whether the more modest aim of empirical adequacy would not provide a better regulatory framework for scientific progress.

Truth also crops up in a completely different context within the philosophy of science: the issue of theory choice, or how best to choose between different theories. For example, in an ideal situation, physicists would be faced with more than one true theory and would then have to pick the one that has independent virtues, such as simplicity and explanatory power. This was the case, for instance, with different formulations of classical mechanics. But since string theory is currently the only contender for a unified theory of physics, researchers are not in such a luxurious position.

logy with Maxwell too seriously one imposes values onto string theory that it need not accept. After all, since string theory aims to unify the basic interactions, its success in spawning new empirical applications or technologies is quite irrelevant. However, renouncing the value of applicability comes at a price, since we do not want a theory that neither tells us how the world really is nor has any interesting applications.

The question of how progressive string theory is then becomes one of truth, and this brings us back to predictions. The more numerous, varied, precise and novel a theory's successful predictions are, the more confidence we can have that the theory is true, or at least approximately true (see box). That a theory describes the world correctly wherever we have checked provides good reason to expect that it will describe the world correctly where we have not checked. String theory's failure to make testable predictions therefore leaves us with little reason to believe that it gives us a true picture.

\section{The appeal of simplicity}

Some philosophers, and physicists alike, argue that empirical tests are not the only route to truth: other dimensions of progress also have a connection with truth, albeit a less direct one. Simplicity is one example. If we assume that we somehow know that the world is simple in a certain way (for example in that it contains only one fundamental entity such as strings), then, all other things being equal, a theory that is not simple in this way cannot be correct. But if such claims are to affect what theories we judge to be true in science, then they need to be carefully argued and justified. The question is whether or not this is possible.

Because many physicists long for simple and unified theories, they sometimes conclude that the world "just has to be" simple. But modern science demands that claims about the world be justified by appeal to the phenomena in the world, not based on longings. A seemingly more promising strategy to defend simplicity is to perform a loose induction on the history of physics: we have accumulated a great number of hugely successful simple theories, hence the world must be simple.

But it is easy to cite counter cases, such as theoretical condensed-matter physics, where progress has not come about in this way. Indeed, even if such counter cases could be dismissed, it is still hard to properly articulate what kind of simplicity all the successful cases share and to argue that string theory is simple in precisely that way. In short, there is no straightforward argument for the conclusion that the world is simple, which means that claims about a theory's truth based on simplicity as at best inconclusive.

Although string theory has progressed along the dimensions of unifying and explanatory power, this in itself is not sufficient to believe that it gives us a true picture of the world. Hence, as it stands, string theory is not yet progressive because it has made progress only along a few of the many dimensions that matter to a research programme's success.

However, one of the punchlines of Lakatos' methodology of scientific research programmes is that we should treat budding programmes leniently, and string theory therefore deserves to be pursued in the hope that one day it will become progressive. In practice, however, the questions of how much to invest in this effort and what should be sacrificed for that investment still remain.
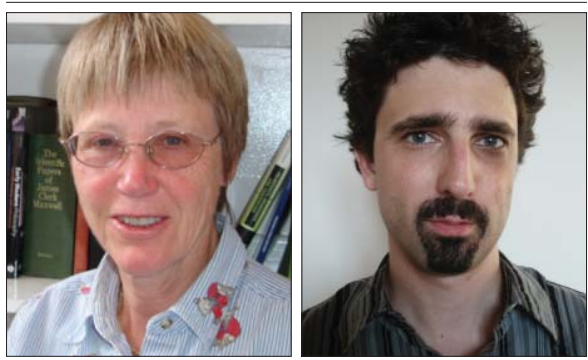

Nancy Cartwright and Roman Frigg are in the Department of Philosophy, Logic and Scientific Method, London School of Economics, UK, e-mail n.I.cartwright@|se.ac.uk 